04

\title{
Особенности структуры и диэлектрических свойств керамик на основе титаната натрия-висмута
}

\author{
(C) Е.Д. Политова ${ }^{1}$, Н.В. Голубко ${ }^{1}$, Г.М. Калева ${ }^{1}$, А.В. Мосунов ${ }^{1}$, Н.В. Садовская ${ }^{1}$, \\ Д.А. Белькова ${ }^{2}$, С.Ю. Стефанович ${ }^{1,2}$ \\ ${ }^{1}$ Научно-исследовательский физико-химический институт им. Л.Я. Карпова, \\ Москва, Россия \\ ${ }^{2}$ Московский государственный университет им. М.В. Ломоносова, \\ Москва, Россия \\ E-mail: politova@cc.nifhi.ac.ru
}

\begin{abstract}
Изучены фазообразование, особенности структуры и диэлектрические свойства керамик составов из области морфотропной фазовой границы в системе $\left(\mathrm{Na}_{0.5} \mathrm{Bi}_{0.5}\right) \mathrm{TiO}_{3}-\mathrm{BaTiO}_{3}$, модифицированных $\mathrm{Bi}\left(\mathrm{Mg}_{0.5} \mathrm{Ti}_{0.5}\right) \mathrm{O}_{3}$, а также легкоплавкими добавками $\mathrm{KCl}, \mathrm{NaCl}-\mathrm{LiF}, \mathrm{CuO}$ и $\mathrm{MnO}_{2}$, способствующими регулированию стехиометрии и свойств керамик. Керамики характеризуются сегнетоэлектрическими фазовыми переходами, проявляющимися в виде скачков при температурах вблизи $400 \mathrm{~K}$ и максимумов при $T_{m} \sim 600 \mathrm{~K}$ на температурных зависимостях диэлектрической проницаемости. Фазовые переходы $\sim 400 \mathrm{~K}$ демонстрируют релаксорное поведение, указывающее на присутствие полярных областей в неполярной матрице. Увеличение содержания $\mathrm{Bi}\left(\mathrm{Mg}_{0.5} \mathrm{Ti}_{0.5}\right) \mathrm{O}_{3}$ способствует понижению электропроводимости и диэлектрических потерь образцов, а относительная диэлектрическая проницаемость при комнатной температуре $\varepsilon_{r t}$ остается на достаточно высоком уровне, достигая наибольших значений $\varepsilon_{r t}=1080-1350$ в керамиках, модифицированных $\mathrm{KCl}$.
\end{abstract}

Работа выполнена при поддержке Российского фонда фундаментальных исследований (проект № 15-03-03269).

DOI: 10.21883/FTT.2018.03.45538.04D

\section{1. Введение}

В настоящее время актуальны исследования, направленные на разработку новых бессвинцовых материалов, функциональные свойства которых не уступают широко используемым материалам на основе цирконататитаната свинца. Оксиды со структурой перовскита на основе сегнетоэлектрика-релаксора титаната натриявисмута $\left(\mathrm{Na}_{0.5} \mathrm{Bi}_{0.5}\right) \mathrm{TiO}_{3}$ (NBT) относятся к наиболее перспективным объектам, так как введением различных добавок можно изменять их свойства в широком диапазоне - от диэлектрических, сегнето- и пьезоэлектрических до ионопроводящих [1-3].

В данной работе изучены фазообразование, особенности структуры и диэлектрические свойства керамик составов из области морфотропной фазовой границы в системе $\left(\mathrm{Na}_{0.5} \mathrm{Bi}_{0.5}\right) \mathrm{TiO}_{3}-\mathrm{BaTiO}_{3}$, модифицированных антисегнетоэлектриком $\mathrm{Bi}\left(\mathrm{Mg}_{0.5} \mathrm{Ti}_{0.5}\right) \mathrm{O}_{3}$, а также легкоплавкими добавками хлорида калия $\mathrm{KCl}, \mathrm{NaCl}-\mathrm{LiF}$, $\mathrm{CuO}$ и $\mathrm{MnO}_{2}$ с целью регулирования стехиометрии и диэлектрических свойств керамических образцов.

\section{2. Экспериментальная часть}

Керамические образцы $(1-y)\left[(1-x)\left(\mathrm{Na}_{0.5} \mathrm{Bi}_{0.5}\right) \mathrm{TiO}_{3}\right.$ $\left.-x \mathrm{BaTiO}_{3}\right]-y \mathrm{Bi}\left(\mathrm{Mg}_{0.5} \mathrm{Ti}_{0.5}\right) \mathrm{O}_{3}($ NBT-BT-MBT) c $x, y=$ $=0.0-0.2$ и образцы $(1-y)\left[0.8\left(\mathrm{Na}_{0.5} \mathrm{Bi}_{0.5}\right) \mathrm{TiO}_{3}\right.$ $\left.-0.2 \mathrm{BaTiO}_{3}\right]-y \mathrm{KCl}(\mathrm{NBT}-\mathrm{BT}-\mathrm{KCl})$ с $y=0-0.15$ и получали методом твердофазного синтеза двух- и/или трехкратным обжигом при температурах $T_{1,2}=$
$=973-1173 \mathrm{~K} \quad(6 \mathrm{~h}), \quad T_{3}=1373-1473 \mathrm{~K} \quad(1-2 \mathrm{~h})$. В качестве исходных реагентов использовали карбонаты $\mathrm{BaCO}_{3}, \mathrm{MgCO}_{3}$ и $\mathrm{Na}_{2} \mathrm{CO}_{3}$ (analytical grade), оксиды $\mathrm{Bi}_{2} \mathrm{O}_{3}, \mathrm{TiO}_{2}, \mathrm{CuO}$ и $\mathrm{MnO}_{2}$ (special purity grade), хлориды $\mathrm{KCl}$ и $\mathrm{NaCl}$ (pure). После синтеза при $T_{2}=900$ $(6 \mathrm{~h})$ к образцам $0.8(0.9 \mathrm{NBT} 0.1 \mathrm{BT})-0.2 \mathrm{BMT}$ добавляли по 2 w.\% (0.6 NaCl-0.4LiF) и по 1 w.\% $\mathrm{CuO}$ и $\mathrm{MnO}_{2}$.

Фазовый состав и параметры кристаллической структуры исследовали при комнатной температуре методом рентгенофазового анализа (РФА) (дифрактометр ДРОН-3М, CuK $K_{\alpha}$-излучение), микроструктуру и элементный анализ образцов - с помощью метода сканирующей электронной микроскопии (СЭМ) (микроскоп JEOL YSM-7401F), диэлектрические свойства керамик - методом диэлектрической спектроскопии (измеритель Agilent 4284 A, 1 V) в интервале температур $300-1000 \mathrm{~K}$ и частот $100 \mathrm{~Hz}-1 \mathrm{MHz}$. Величину спонтанной поляризации образцов оценивали, используя результаты измерений методом генерации второй гармоники лазерного излучения (ГВГ) ( $\mathrm{Nd}: \mathrm{YAG}$ лазер, $\lambda=1.064 \mu \mathrm{m})$.

\section{3. Результаты и обсуждение}

Согласно данным РФА, образцы с псевдокубической структурой перовскита формируются при температурах $T_{1}=973-1173 \mathrm{~K}$. Плотные однофазные образцы были получены после обжига модифицированных образцов при $T_{3}=1373-1473 \mathrm{~K}$. Смещение положения дифракционных пиков в область меньших углов (рис. 1) свидетельствует об увеличении объема псевдокубической 

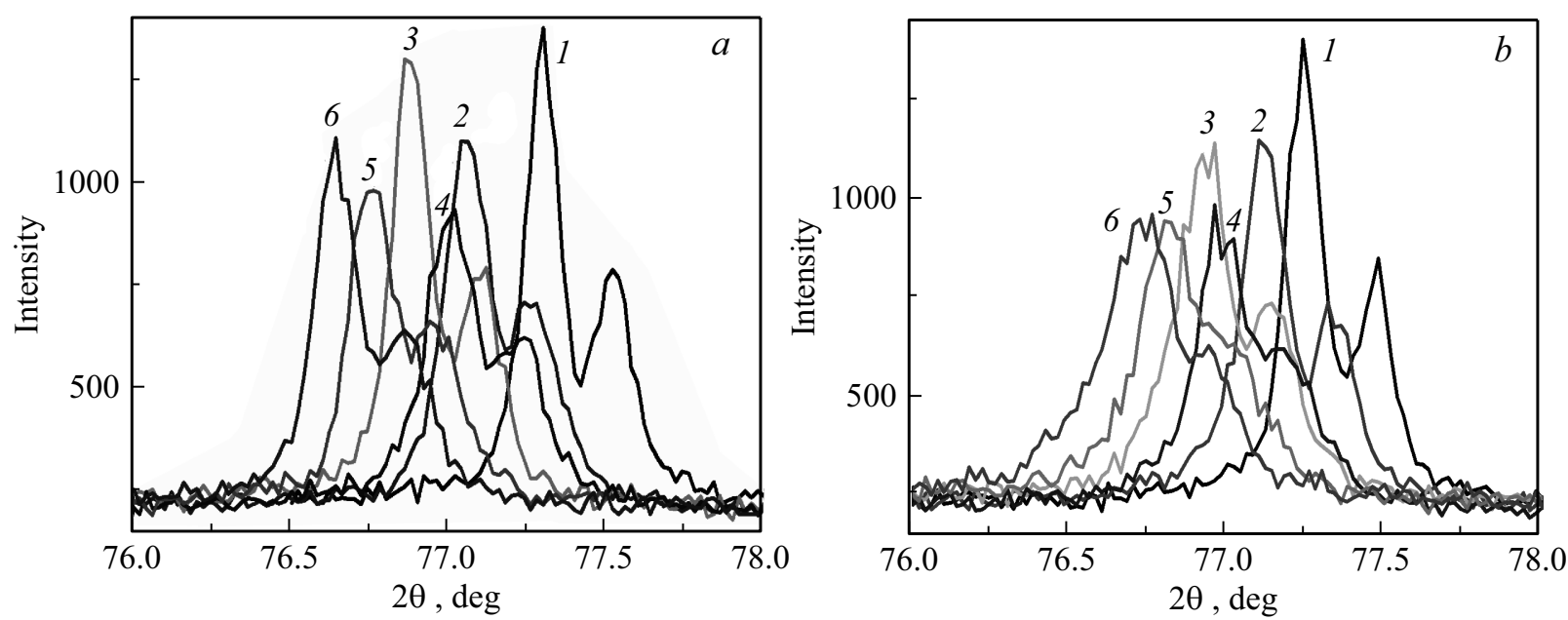

Рис. 1. Участки дифрактограмм, соответствующие дифракционным пикам с $h^{2}+k^{2}+l^{2}=10$, модифицированных образцов системы $(1-y)\left[(1-x)\left(\mathrm{Na}_{0.5} \mathrm{Bi}_{0.5}\right) \mathrm{TiO}_{3}-x \mathrm{BaTiO}_{3}\right]-y \mathrm{Bi}\left(\mathrm{Mg}_{0.5} \mathrm{Ti}_{0.5}\right) \mathrm{O}_{3}$ с $x=0.05(1-3)$ и $0.10(4-6), y=0 \quad(1,4), 0.1(2,5)$, $0.2(3,6)(a)$, модифицированных образцов $(1-y)\left[(1-x)\left(\mathrm{Na}_{0.5} \mathrm{Bi}_{0.5}\right) \mathrm{TiO}_{3}-x \mathrm{BaTiO}_{3}\right]-y \mathrm{Bi}_{\left(\mathrm{Mg}_{0.5} \mathrm{Ti}_{0.5}\right) \mathrm{O}_{3}} \quad \mathrm{c} \quad x=0.05 \quad(1-3)$ и $0.10(4-6), y=0(1,4), 0.1(2,5), 0.2(3)(b)$.

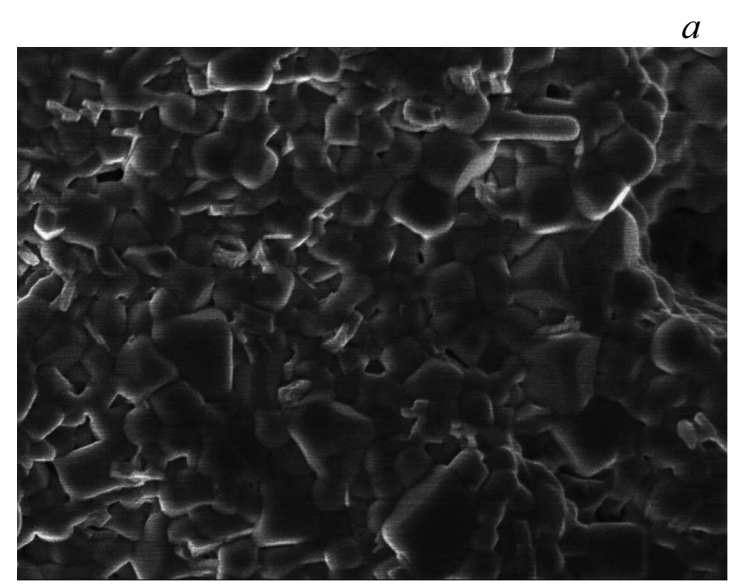

$-1 \mu \mathrm{m}$

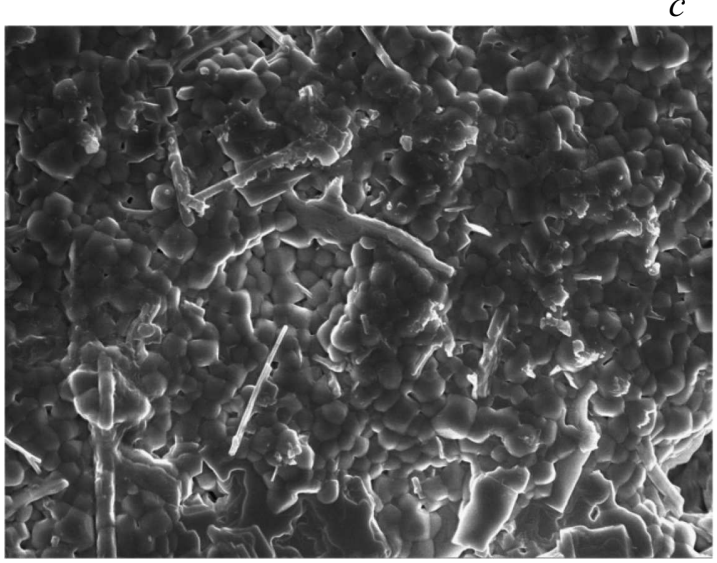

$10 \mu \mathrm{m}$

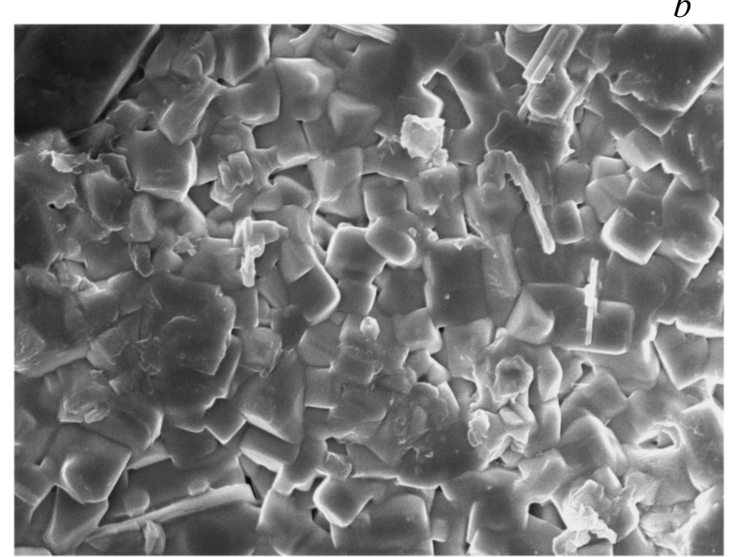

$-1 \mu \mathrm{m}$

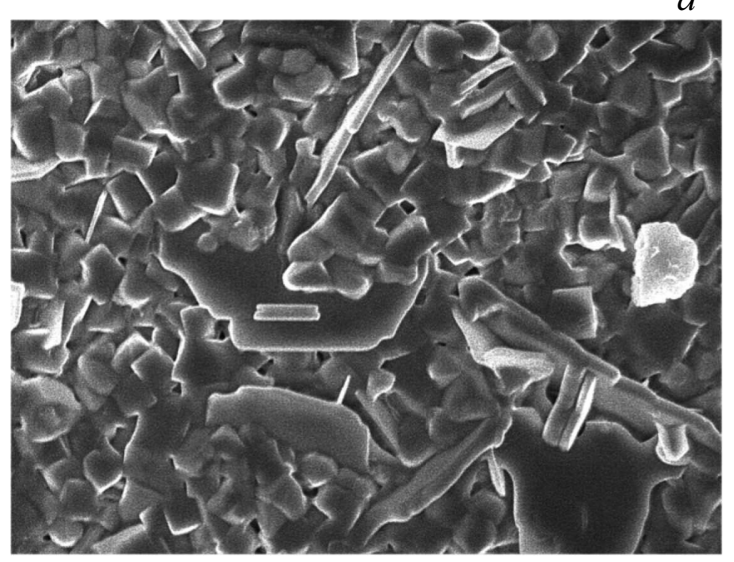

$10 \mu \mathrm{m}$

Рис. 2. Микрофотографии поверхности модифицированных образцов керамик $(1-y)\left[(1-x)\left(\mathrm{Na}_{0.5} \mathrm{Bi}_{0.5}\right) \mathrm{TiO}_{3}-x \mathrm{BaTiO}_{3}\right]$ $-y \mathrm{Bi}\left(\mathrm{Mg}_{0.5} \mathrm{Ti}_{0.5}\right) \mathrm{O}_{3}$ с $x=0.05, y=0(a), x=0.05, y=0.2(b), x=0.1, y=0.1(c), x=0.1, y=0.2(d)$. Масштабные полоски: $1 \mu \mathrm{m}(a, b)$ и $10 \mu \mathrm{m}(c, d)$. Образцы получены при $T_{1}=973 \mathrm{~K}, T_{2}=1173 \mathrm{~K}(6 \mathrm{~h}), T_{3}=1373 \mathrm{~K}(1 \mathrm{~h})$. 

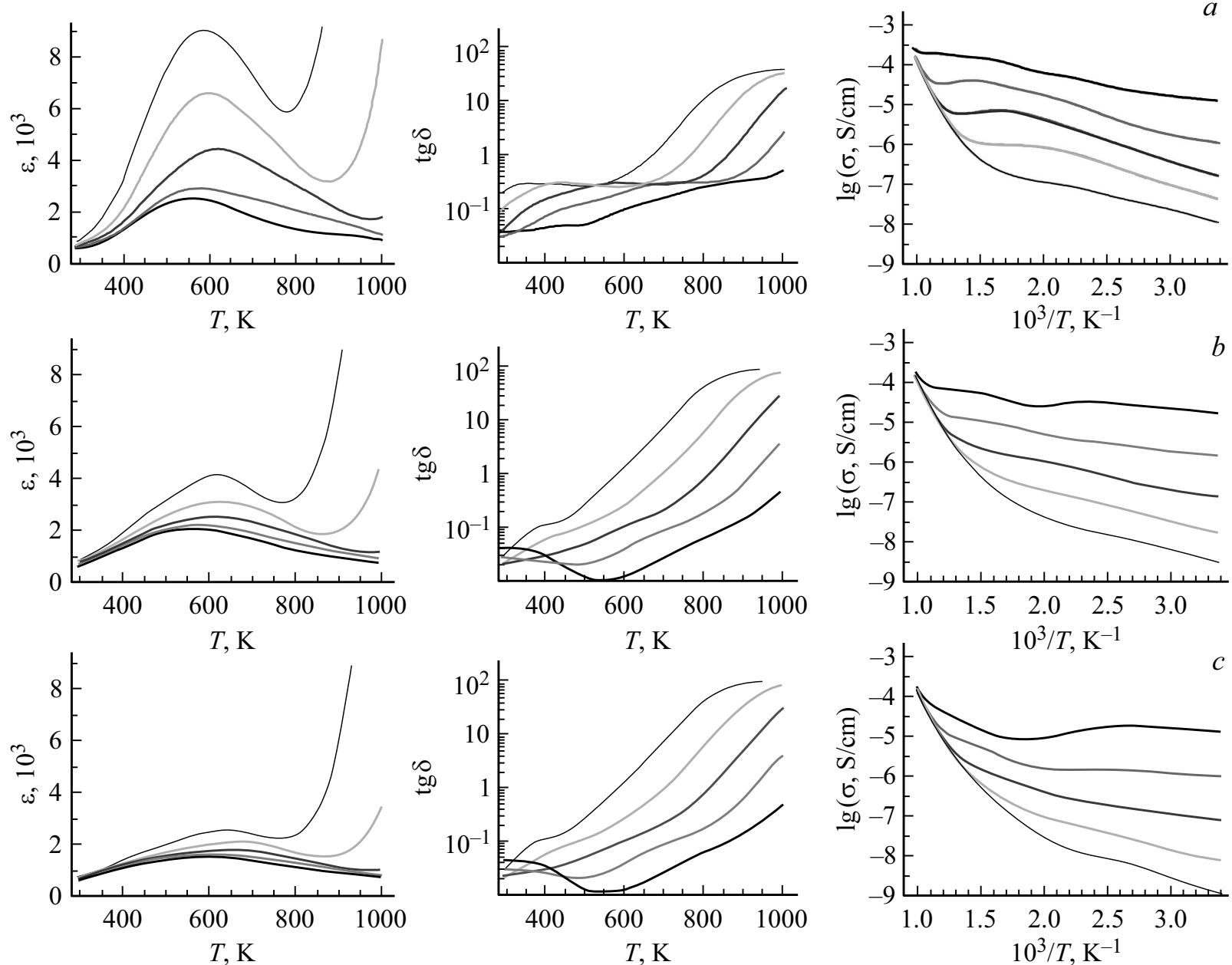

Рис. 3. Температурные зависимости диэлектрической проницаемости $\varepsilon(T)$, тангенса угла диэлектрических потерь $\operatorname{tg} \delta(T)$ и логарифма электропроводимости $1 / 1000 \lg \sigma(1 / T)$ модифицированных керамик $(1-y)\left[(1-x)\left(\mathrm{Na}_{0.5} \mathrm{Bi}_{0.5}\right) \mathrm{TiO}_{3}-x \mathrm{BaTiO}_{3}\right]$ $-y \mathrm{Bi}\left(\mathrm{Mg}_{0.5} \mathrm{Ti}_{0.5}\right) \mathrm{O}_{3}$ с $x=0.05(a-c), 0.10(d-f), y=0(a, d), 0.1(d, e)$ и $0.2(c, f)$, измеренных на частотах $f=100 \mathrm{~Hz}, 1,10$, $100 \mathrm{kHz}, 1 \mathrm{MHz}$. Образцы получены при $T_{1}=973, T_{2}=1173(6 \mathrm{~h}), T_{3}=1373 \mathrm{~K}(1 \mathrm{~h})$.

ячейки перовскита в результате замещения катионов A- $\left(\mathrm{Na}^{1+}\right.$ и $\left.\mathrm{Bi}^{3+}\right)$ и В- $\left(\mathrm{Ti}^{4+}\right)$ катионами с большими ионными радиусами $\left(\mathrm{Ba}^{2+}, \mathrm{K}^{1+}\right.$ и $\mathrm{Mg}^{2+}$ соответственно). Наблюдаемое уширение пиков на дифрактограммах образцов с $x=0.2$ свидетельствует о формировании тетрагональной симметрии решетки образцов NBT-BT с $x=0.2, y=0$ в соответствии с данными работ [4-6]. Добавление оксида ВМТ и хлорида $\mathrm{KCl}$ стимулируют формирование псевдокубической решетки.

Микроструктура модифицированных образцов NBTBT-MBT и NBT-BT-KCl характеризуется изометричными зернами размером $\sim 1-5 \mu \mathrm{m}$ (рис. 2 ). Согласно данным элементного анализа, катионы меди входят в решетку основной фазы.

Температурные зависимости диэлектрической проницаемости характеризуются слабо выраженными аномалиями при температурах $\sim 400 \mathrm{~K}$ и выраженными максимумами при $T_{m} \sim 580-610 \mathrm{~K}$ (рис. 3,4 ). При температуpax выше $\sim 700 \mathrm{~K}$ выявлены эффекты диэлектрической релаксации, указывающие на присутствие вакансий в подрешетке кислорода.
Фазовые переходы вблизи $400 \mathrm{~K}$ демонстрируют выраженное релаксорное поведение, указывающее на присутствие полярных областей в неполярной матрице. Это подтверждается данными ГВГ и согласуется с рассмотрением составов $\mathrm{NBT}-\mathrm{BaTiO}_{3}$ из области МФГ как состоящих из сегнетоэлектрических кластеров, внедренных в неполярную матрицу [7,8]. Нами установлено, что при комнатной температуре интенсивность сигнала ГВГ $q$ образцов NBT-BТ изменяется от $q \approx 6(x=0.05)$ до $q \approx 50 \quad(x=0.2)$ [9]. Эти значения существенно выше значений $I_{2 \omega}\left(\mathrm{SiO}_{2}\right)$ для стандартного порошка $\alpha-\mathrm{SiO}_{2}$ и подтверждают, таким образом, нецентросимметричную структуру оксидов. С другой стороны, эти значения $q$ на 2 порядка ниже сигнала ГВГ, типичного для сегнетоэлектриков, что соответствует природе МФГ составов NBT-BT, рассматриваемых как состоящих из сегнетоэлектрических ромбоэдрических кластеров, внедренных в неполярную тетрагональную матрицу, существующую в широком интервале температур вплоть до $\sim 800 \mathrm{~K}[7,8]$. Зарядовый беспорядок в А-позициях, занимаемых случайным образом распределенными раз- 

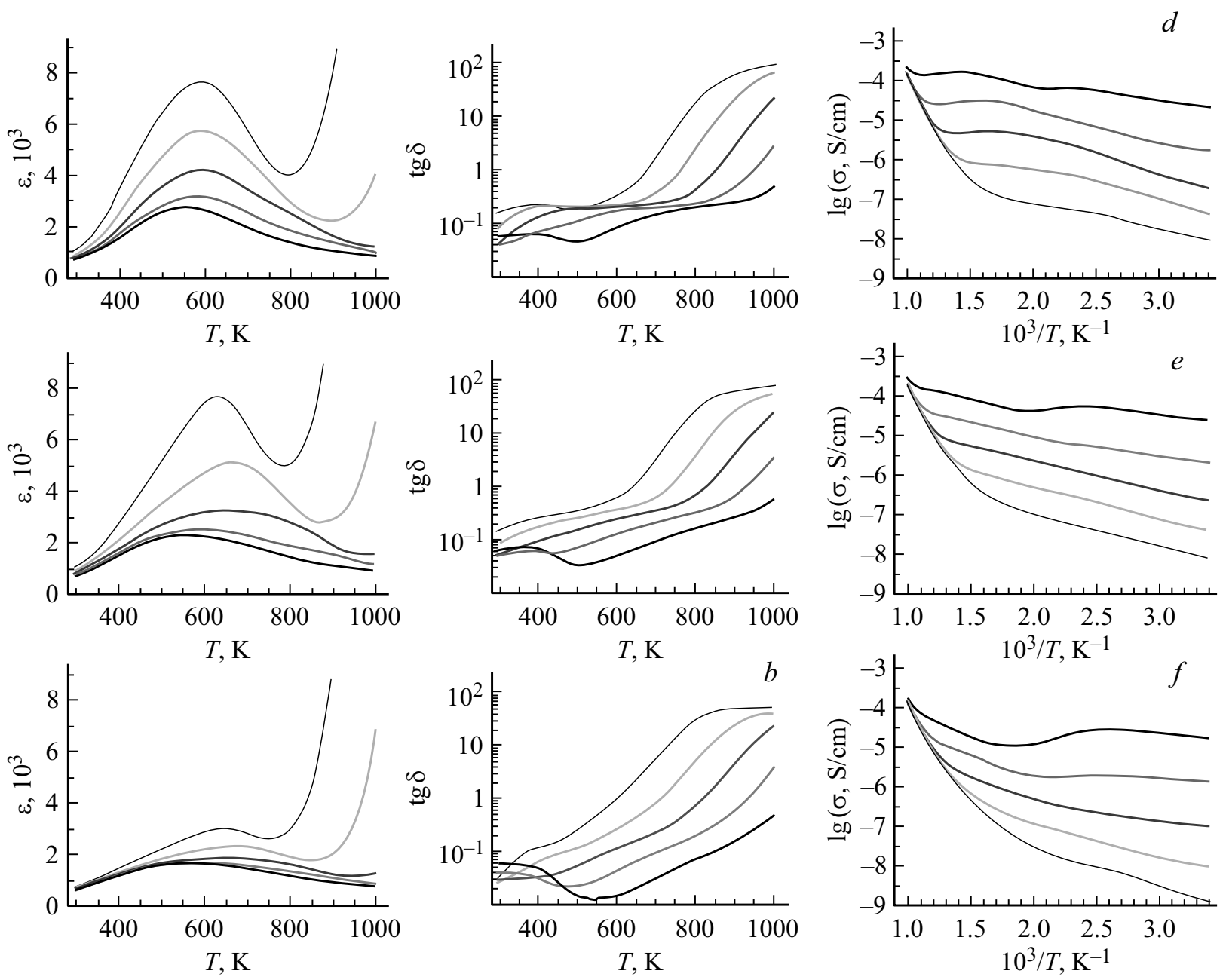

Рис. 3 (продолжение).
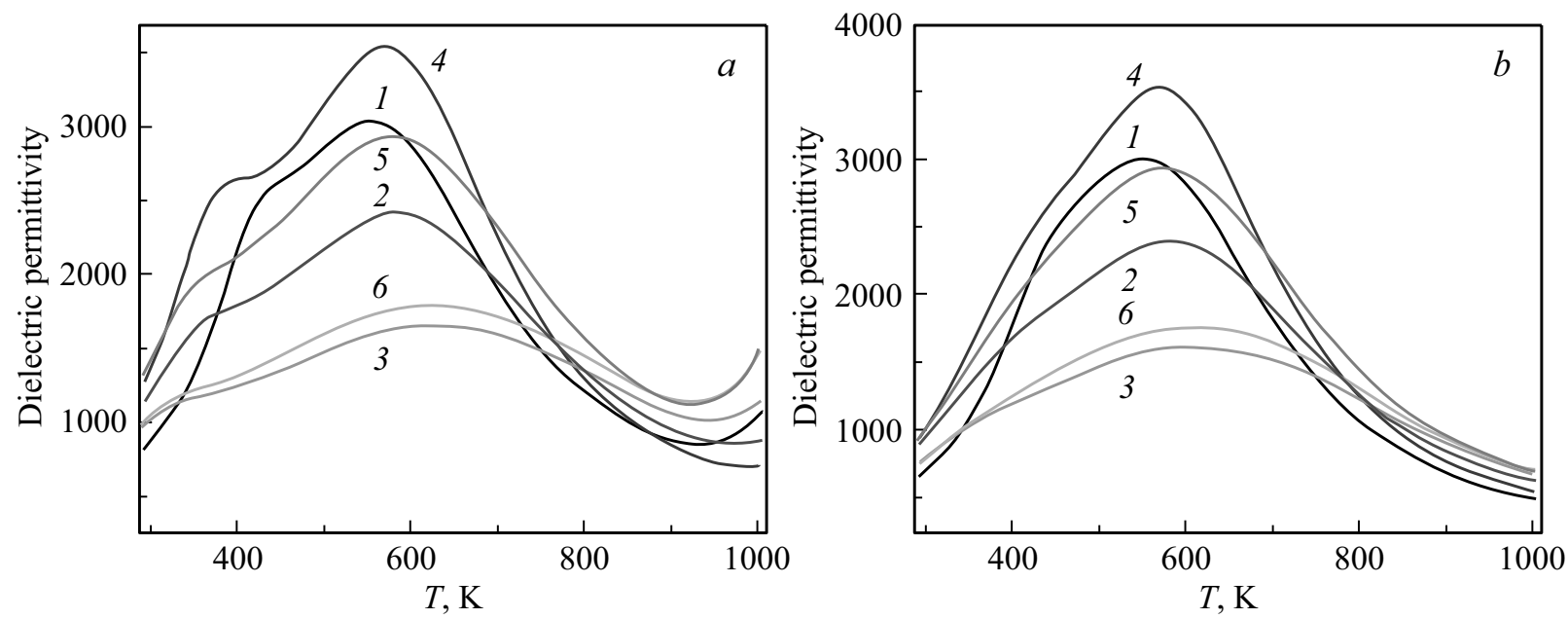

Рис. 4. Температурные зависимости диэлектрической проницаемости $\varepsilon(T)$ модифицированных образцов $(1-y)\left[(1-x)\left(\mathrm{Na}_{0.5} \mathrm{Bi}_{0.5}\right) \mathrm{TiO}_{3}-x \mathrm{BaTiO}_{3}\right]-y \mathrm{Bi}\left(\mathrm{Mg}_{0.5} \mathrm{Ti}_{0.5}\right) \mathrm{O}_{3}$ с $x=0.05$ (1-3) и 0.10 (4-6), $y=0 \quad(1,4), 0.1 \quad(2,5)$ и $0.2(3,6)$, измеренных на частотах $f=1 \mathrm{kHz}(a)$ и $1 \mathrm{MHz}(b)$. 
нозарядовыми катионами $\left(\mathrm{Bi}^{3+}, \mathrm{Na}^{+}, \mathrm{Ba}^{2+}, \mathrm{K}^{+}\right.$и $\left.\mathrm{Li}^{+}\right)$, приводит к формированию случайных электрических полей, влияющих на локальный баланс между смещениями из центральных позиций катионов в позициях В и А решетки перовскита, способствуя локальным корреляциям диполей и формированию релаксорных свойств образцов [8].

Электропроводимость и диэлектрические потери образцов, измеренные при комнатной температуре, уменьшаются при увеличении содержания МВТ, тогда как относительная диэлектрическая проницаемость при комнатной температуре $\varepsilon_{r t}$ остается на достаточно высоком уровне $\left(\varepsilon_{r t}=700-1200\right.$ при $\left.f=1 \mathrm{kHz}\right)$ для образцов NBT-BT-BMT c $x=0.05, y=0-0.2 ; x=0.1, y=0.1$ и $0.2, x=0.2, y=0.2$. Эти образцы имеют сравнительно низкие диэлектрические потери (3.6-5\%). Керамики NBT-BT, модифицированные $\mathrm{KCl}$, характеризуются наибольшими значениями $\varepsilon_{r t}=1080-1350$ при значениях $\operatorname{tg} \delta=7.4-8 \%$ при $x=0.0-0.075$. Их электропроводимость изменяется немонотонным образом: немного повышается при $y=0-0.075$, затем понижается при $y>0.075$, указывая на уменьшение количества вакансий в позициях А и в подрешетке кислорода.

\section{4. Заключение}

Изучены особенности фазообразования, параметры структуры, микроструктура и диэлектрические свойства керамик составов из области морфотропной фазовой границы в системе $\left(\mathrm{Na}_{0.5} \mathrm{Bi}_{0.5}\right) \mathrm{TiO}_{3}-\mathrm{BaTiO}_{3}$, модифицированных $\mathrm{Bi}\left(\mathrm{Mg}_{0.5} \mathrm{Ti}_{0.5}\right) \mathrm{O}_{3}$, а также легкоплавкими добавками $\mathrm{KCl}, \mathrm{NaCl}-\mathrm{LiF}, \mathrm{CuO}$ и $\mathrm{MnO}_{2}$. Полученные результаты свидетельствуют об улучшении диэлектрических характеристик изученных керамик, модифицированных гетеровалентными добавками по катионным подрешеткам А и В.

\section{Список литературы}

[1] S.V. Vakhrushev, V.A. Isupov, B.E. Kvyatkovsky, N.M. Okuneva, I.P. Pronin, G.A. Smolensky, P.P. Syrnikov. Ferroelectrics 63, 153 (1985).

[2] P.K. Panda. J. Mater. Sci. 44, 5049 (2009).

[3] J. Rodel, K.G. Webber, R. Dittmer, Wook Jo, M. Kimura, D. Damjanovic. J. Euro. Ceram. Soc. 35, 1659 (2015).

[4] T. Takenaka, K. Maruyama, K. Sakata. Jpn. J. Appl. Phys. 30, 2236 (1991).

[5] C. Ma, X. Tan, E. Dul'kin, M. Roth. J. Appl. Phys. 108, 104105 (2010).

[6] F. Cordero, F. Craciun, F. Trequattrini, E. Mercadelli, C. Galassi. Phys. Rev. B 81, 144124 (2010).

[7] V.V. Shvartsman, D.C. Lupascu. J. Am. Ceram. Soc. 95, 1 (2012).

[8] W. Kleemann. Int. J. Mod. Phys. B 7, 2469 (1993).

[9] E.D. Politova, N.V. Golubko, G.M. Kaleva, A.V. Mosunov, N.V. Sadovskaya, D.A. Belkova, S.Yu. Stefanovich. Proceedings of the 2017 Joint IEEE/ISAF/IWATMD/PFM Symposium, Atlanta, Georgia, USA, 7-11 May 2017, p. 75. 